\title{
Molecular genetics for familial hypercholesterolemia
}

\author{
Nicola Marziliano ${ }^{1,2,3, *}$, Alessandro Medoro ${ }^{1}$, Stefano Folzani $^{3}$, Mariano Intrieri ${ }^{1}$, \\ Claudio Reverberi ${ }^{3}$
}

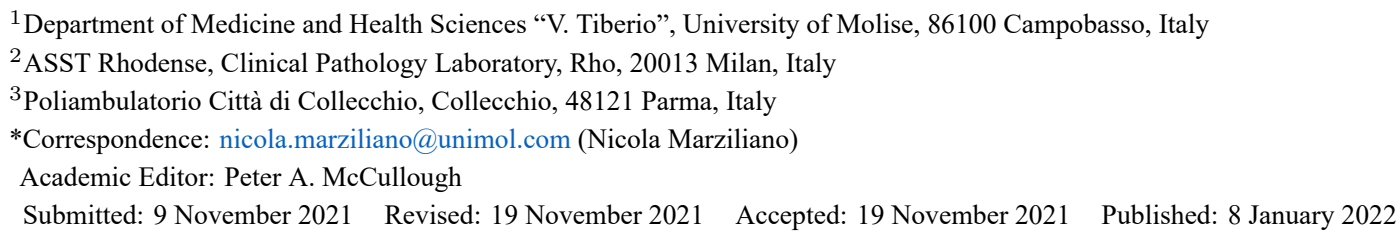

Familial Hypercolesterolemia (FH; MIM 143890 and 603813) is a group of inherited genetic defects characterized by abnormal plasma levels of Low-Density Lipoprotein (LDL) cholesterol $(>190 \mathrm{mg} / \mathrm{dL})$ that are correlated with increased risk for coronary artery disease (CAD) [1,2]. Most FH show autosomal dominant mutations in LowDensity Lipoprotein Receptor $(L D L R)$; additionally also mutations in other genes coding for proteins involved in cholesterol metabolism or LDLR processing and function, such as Proprotein Convertase Subtilisin/Kexin Type 9 (PCSK9) and Apolipoprotein B (APOB), can underly the same clinical condition, although less frequently [3]. Mutations in the gene encoding LDL Receptor Adaptor Protein 1 (LDLRAP1) produce a very rare autosomal recessive with a similar phenotypic expression [4]. Genetic variants that lower the expression levels of the above-mentioned genes increase the risk of coronary heart disease, thus being the ground for the development of CAD. Consequently, identifying such genetic variants has emerged as a therapeutic strategy to reduce the risk of CAD both in primary and secondary prevention in patients experiencing myocardial infarction (MI) $[5,6]$.

Beyond identifying a therapeutic and druggable target gene, human genetics could help to evaluate the probable efficacy and safety of the pharmacologic modulation. Previous works addressing the diagnostic yield of genetic testing in FH have focused on subjects with FH clinically ascertained or suspected; they showed that the FH mutation prevalence has slightly wide ranged (from $20 \%$ to $80 \%$ $[7,8])$. This aspect might be due to different ascertainment of the phenotypes by making use of family history, elevated LDL cholesterol at a young age, physical examination features or referral to specialized clinics, each of which may enrich for monogenic causes. Of note, if the ascertainment from the general population is made solely on the serum LDL cholesterol levels, the extent to which FH mutations contribute to severe hypercholesterolemia is largely unknown. Such knowledge may inform the design and effectiveness of universal general population FH screening proposals.

Nowadays, molecular genetics is far from being performed on an ivory tower but -vice versa- throughout new chemistries and new sequencing platforms can be easily incorporated into clinical pathology laboratories' workflow especially for diseases such as FH and oncology (both inherited and acquired) [8,9].

We analyzed by means of Next Generations Sequencing (NGS) four FH genes (LDLR, APOB, PCSK9 and $L D L R A P 1$ ), in our single referral center (Parma hinterland, Italy) for more than 10 years enrolling 3025 consecutive participants both for primary (LDL cholesterol $\geq 190$ $\mathrm{mg} / \mathrm{dL}$ ) and secondary (after experiencing MI) prevention, to determine: (1) the diagnostic yield of FH mutation detection in participants with increased LDL serum levels; and (2) the clinical impact of any FH mutation on CAD risk within any given stratum of LDL cholesterol levels.

Among 3025 caucasians participants, we found that a considerable fraction (757 patients; 25.05\%) carried an FH mutation. Moreover, patients who carried an FH mutation were at substantially higher risk for CAD than those who did not for each stratum of LDL cholesterol. This increased CAD risk among mutation carriers can be explained partially by a greater cumulative lifetime exposure to raised LDL cholesterol.

These results permit the following conclusions: (a) when participants were solely ascertained for abnormal LDL cholesterol level $(>190 \mathrm{mg} / \mathrm{dL})$, we identified an FH mutation in $25.05 \%$ of hypercholesterolemic patients. These sequencing yelds are partially overlapping with other previous screening studies [7,8]; (b) CAD risk was higher in patients with an FH mutation than in those without for any LDL cholesterol level. Taking together, these facts shed additional lights on the actual utility of genetic testing to provide risk information beyond the laboratory data of the LDL cholesterol level. These data support the hypothesis that an FH mutation, present since birth, increases CAD risk via a lifelong and continuous exposure to high LDL cholesterol [10].

Finally, these data might also provide insights on how to clinically define FH. In a consolidated fashion, FH refers to elevated LDL cholesterol with an underlying mutation/s in any of several genes segregating in an autosomal dominant/recessive manner. Differnet approaches that incorporate two features, LDL cholesterol threshold and muta- 
tion definition, affect $\mathrm{FH}$ prevalence estimates. By solely making use of untreated LDL cholesterol $\geq 190 \mathrm{mg} / \mathrm{dL}$ (i.e., without an $\mathrm{FH}$ mutation requirement) approximately $7 \%$ of the U.S. adult population would be classified as being affected by FH thus combining non genetic and genetic causes [11].

In the case of routine genetic testing is not available, several clinical scoring systems, such as Simon Broome, Dutch Lipid Clinical Network, and MEDPED criteria, have been developed to approximate FH status [5]. However, we strongly support the incorporation of NGS into a laboratory workflow for several reasons: (a) can be easily automated; (b) costs are dropping down; (c) analysis algorithms are automated too. We are not minimizing the presence of genetics laboratories, on the contrary because genetics is a powerful tool, it should be handled in a more generalized laboratory setting when it comes to common diseases such as FH to improve patients' diagnosis and prognosis.

The primary goal of precision medicine is the use of molecular diagnostics to identify those individuals within a given population at increased CAD risk and in which to deliver a tailored intervention. Systematic efforts-molecular genetics included - to identify and treat severely hypercholesterolemic patients who carry an FH mutation could represent one such opportunity.

\section{Author contributions}

$\mathrm{NM}, \mathrm{AM}$ conceived and wrote the manuscript; SF, CR provided clinical cases and criticized the manuscript; $\mathrm{MI}$ supervised the writing activities. All authors read and approved the final manuscript.

\section{Ethics approval and consent to participate}

Not applicable.

\section{Acknowledgment}

We would like to express our gratitude to all those who helped us during the writing of this manuscript.

\section{Funding}

This research was funded by ASI SUTURE project, grant number DC-WM-7016-068.

\section{Conflict of interest}

The authors declare no conflict of interest.

\section{References}

[1] Aggarwal DJ, Kathariya MG, Verma DPK. LDL-C, NON-HDL$\mathrm{C}$ and $\mathrm{APO}-\mathrm{B}$ for cardiovascular risk assessment: Looking for the ideal marker. Indian Heart Journal. 2021; 73: 544-548.

[2] Roberts R, Fair J. Genetics, its role in preventing the pandemic of coronary artery disease. Clinical Cardiology. 2021; 44: 771779 .

[3] Brandts J, Ray KK. Familial Hypercholesterolemia. Journal of the American College of Cardiology. 2021; 78: 1831-1843.

[4] Alonso R, Díaz-Díaz JL, Arrieta F, Fuentes-Jiménez F, de Andrés R, Saenz P, et al. Clinical and molecular characteristics of homozygous familial hypercholesterolemia patients: Insights from SAFEHEART registry. Journal of Clinical Lipidology. 2016; 10: 953-961.

[5] Stone NJ, Robinson JG, Lichtenstein AH, Bairey Merz CN, Blum CB, Eckel RH, et al. 2013 ACC/AHA guideline on the treatment of blood cholesterol to reduce atherosclerotic cardiovascular risk in adults: a report of the American College of Cardiology/American Heart Association Task Force on Practice Guidelines. Circulation. 2014; 129: S1-45.

[6] Gidding SS, Ann Champagne M, de Ferranti SD, Defesche J, Ito MK, Knowles JW, et al. The Agenda for Familial Hypercholesterolemia. Circulation. 2015; 132: 2167-2192.

[7] Lombardi MP, Redeker EJW, Gent DHMV, Smeele KL, Weerdesteijn R, Mannens MMAM. Molecular Genetic Testing for Familial Hypercholesterolemia in the Netherlands: a Stepwise Screening Strategy Enhances the Mutation Detection Rate. Genetic Testing. 2006; 10: 77-84.

[8] Taylor A, Wang D, Patel K, Whittall R, Wood G, Farrer M, et al. Mutation detection rate and spectrum in familial hypercholesterolaemia patients in the UK pilot cascade project. Clinical Genetics. 2010; 77: 572-580.

[9] Hicks JK, Howard R, Reisman P, Adashek JJ, Fields KK, Gray $\mathrm{JE}$, et al. Integrating Somatic and Germline next-Generation Sequencing into Routine Clinical Oncology Practice. JCO Precision Oncology. 2021; 5: 884-895.

[10] Brown MS, Goldstein JL. Biomedicine. Lowering LDL-not only how low, but how long? Science. 2006; 311: 1721-1723.

[11] de Ferranti SD, Rodday AM, Mendelson MM, Wong JB, Leslie LK, Sheldrick RC. Prevalence of Familial Hypercholesterolemia in the 1999 to 2012 United States National Health and Nutrition Examination Surveys (NHANES). Circulation. 2016; 133: 1067-1072. 